HIP-2002-35/TH

\title{
A scheme with two large extra dimensions confronted with neutrino physics
}

\author{
J. Maalampi ${ }^{1,2 *}$, V. Sipiläinen ${ }^{3 \dagger}$ and I. Vilja ${ }^{4 \dagger}$ \\ ${ }^{1}$ Department of Physics, University of Jyväskylä, Finland \\ ${ }^{2}$ Helsinki Institute of Physics, FIN-00014 University of Helsinki, Finland \\ ${ }^{3}$ Department of Physics, FIN-00014 University of Helsinki, Finland \\ ${ }^{4}$ Department of Physics, FIN-20014 University of Turku, Finland
}

November 25, 2002

\begin{abstract}
We investigate a particle physics model in a six-dimensional spacetime, where two extra dimensions form a torus. Particles with Standard Model charges are confined by interactions with a scalar field to four four-dimensional branes, two vortices accommodating ordinary type fermions and two antivortices accommodating mirror fermions. We investigate the phenomenological implications of this multibrane structure by confronting the model with neutrino physics data.
\end{abstract}

*jukka.maalampi@phys.jyu.fi

†ville.sipilainen@helsinki.fi

${ }_{\ddagger}^{\ddagger}$ vilja@utu.fi 


\section{Introduction}

The idea that the physical spacetime might have more than three spatial dimensions dates back to the beginning of the 20th century and to the attempts of Nordström, Kaluza and Klein to unify gravity and electromagnetism [1]. These early ideas have been resurrected in the present-day string theories which unify gauge theories and gravity in a ten or eleven dimensional spacetime. Extra dimensions were originally thought to be compactified to extremely short distances. However, according to some new ideas developed within the string theories some of them could be relatively large and could have phenomenological consequences testable in physics experiments.

It has been realized that the presence of large extra compact spatial dimensions could make the fundamental Planck scale $M_{*}$, that is the Planck scale associated with the higher-dimensional spacetime, much smaller than it is in the ordinary four-dimensional world [2]-4]. This would offer an attractive solution to the hierarchy problem, i.e. the disparity of the fundamental energy scales of weak interactions and gravity. The weakness of the gravity as seen in our visible four-dimensional spacetime, or in other words the largeness of the effective four-dimensional Planck scale $M_{P l} \simeq 10^{19} \mathrm{GeV}$, could be understood to follow from the spreading of the gravity force lines into the extra dimensions. Gravitational interactions have not yet been tested at short distances, and at present experiments still allow some of these extra dimensions to be as large as tenths of a millimeter [5, 6]. In order to have the Planck scale $M_{*}$ at the TeV range, as the solution of the hierarchy problem requires, at least two extra dimensions are necessarily needed [2].

The basic assumption of the extra dimension scenario is that all the fields charged under the Standard Model (SM) gauge group are localized on a brane, the familiar 3+1dimensional space-time, embedded in the $4+n$-dimensional space, called the bulk [2]. This is not only true for the SM non-singlet fermions and scalars, but also for the SM gauge bosons, which are attached to the branes e.g. due to a gauge trapping mechanism [3]. In string theory the SM particles correspond to open strings whose ends are stuck to the branes, while gravitons and other particles that do not carry SM charges correspond to closed strings free to propagate in the extra dimensions (see e.g. Refs. [3] [7, 8]). 
The possible unlocalizability of right-handed neutrinos $\left(\nu_{R}\right)$ in extra-dimension scenarios was emphasized in [9, 10]. This feature may turn out to be of crucial importance from the neutrino physics point of view. The Yukawa coupling between the left-handed neutrino $\nu_{L}$, residing on the brane, and the right-handed neutrino $\nu_{R}$, residing wherever in the spacetime, is suppressed by a factor of the order of $M_{*} / M_{P l}$ due to the scarce overlapping of the corresponding wave functions. This would offer a new and elegant explanation for the lightness of neutrinos. Perhaps the most popular conventional explanation for the lightness of neutrinos is given by the seesaw mechanism, which requires the existence of a new mass scale around $10^{12} \mathrm{GeV}$ or higher. In the extra-dimension scheme such a high mass scale is neither needed nor naturally appears but small neutrino masses follow from the suppression of the Yukawa couplings due to the large bulk volume.

The possible existence of bulk neutrinos implies another novel feature. The momenta of bulk fields are quantized in the compactified extra dimensions, and each of these quantum states corresponds to a massive state, a Kaluza-Klein excitation, at the brane world. Hence a bulk neutrino would appear as an infinite tower of massive Dirac neutrinos in our four-dimensional spacetime. These new degrees of freedom would hugely enrich the neutrino mass spectrum and neutrino oscillation patterns, as discussed in many recent papers, see e.g. [11].

In our previous work [12] we investigated a model with one large extra dimension paying a particular attention to the effects of the extra dimension in neutrino physics. The localization of the SM fermions in the four-dimensional space was achieved by introducing a five-dimensional scalar field with position dependent expectation value having a domainwall profile in the extra dimension [13, 14. Due to the periodicity of the scalar field in the compactified fifth dimension, there necessarily exists, in addition to the ordinary SM brane, another brane, a mirror brane, in the bulk. We constructed a concrete mathematical framework that appropriately described this two-brane scheme. We extracted a finite neutrino mass matrix, of which the massive Kaluza-Klein modes were integrated out, and studied neutrino masses and mixing from phenomenological point of view. It was found out that both the neutrino properties and the size of the extra dimension, $R$, are in this scheme severely constrained by experimental data. Especially, $R$ turned out to 
be bounded from below due to the upper limit of the electron neutrino mass set by the tritium beta decay experiments. The mixing between the ordinary left-handed neutrino and the inert left-handed bulk neutrino is fairly small if $R \lesssim 10^{-3} \mathrm{~mm}$, but maximal mixing becomes possible for a larger $R$. A large radius, $R \gtrsim 10^{-2} \mathrm{~mm}$, seemed also to be favored by cosmological arguments. Finally, all the active-sterile neutrino mixing schemes considered as possible solutions to the solar and atmospheric neutrino anomalies were also found realizable in this model.

As mentioned before, the hierarchy problem cannot be solved if there is only one extra dimension. In this paper we therefore extend our previous analysis to the case of two extra dimensions. The two-dimensional extra space is assumed to be compactified on a torus. The torus geometry allows for a flat metric, which makes various mathematical manipulations quite straightforward.

We are interested in figuring out in which respect this more realistic six-dimensional scheme differs from our previously studied five-dimensional model. In particular, we would like to find out how the requirement of sufficiently large extra dimensions - so as to solve the hierarchy problem - restricts the parameter space of neutrino physics, that is, mixing angles and squared mass differences.

We will use a similar field-theoretical fermion-trapping mechanism as used in earlier studies [2, 15, 16. We introduce a six-dimensional scalar field that has a vortex-like solution, the field vanishing at the center of the vortex [17]. The SM fermions, assumed to be coupled to the scalar field, are then trapped in the core of the four-dimensional vortex, the brane. The periodicity of the scalar field implies that the vortex must have two vis-à-vis antivortices in both of the extra toroidal dimensions. Consequently, the antivortices require then existence of two vortices, so the vortex configuration consists of two vortices and two antivortices. In other words, besides "our" SM brane one has another brane where particles have usual chiralities and two mirror branes containing mirror particles with chiralities opposite to those of their SM counterparts. In addition to gravitons, only right-handed ordinary neutrinos and their opposite-handed mirror partners are free to propagate in the higher-dimensional bulk. Note that our way of reasoning here is perfectly analogous to the case of one extra dimension [12, except that now the number 
of branes and mirror branes is doubled.

Note that the vortex structure on a sphere would be simpler with just one vortexantivortex pair. However, the curvature of a sphere is non-zero and therefore no fermion zero modes exist on a sphere [18]. As a consequence the bulk (inert) neutrino structure and the interplay between the bulk and the brane (active) neutrinos would be much more complicated.

In the following Section we shall introduce the mathematical framework of our sixdimensional scheme and study its effective action in the four-dimensional spacetime. The neutrino mass matrix is derived and a phenomenological analysis of neutrino physics of the model is performed in Section 3. Section 4 is devoted to a summary and conclusions.

\section{Formulation of the model}

In this section we consider a concrete six-dimensional model that exhibits the feature of localizing the left-handed neutrinos on branes and the right-handed mirror neutrinos on mirror branes while letting their gauge inert opposite-handed companions to live in the entire higher-dimensional bulk. The localization of the gauge active neutrinos is achieved by the field-theoretical method that was discussed in e.g. Ref. [2] and that we applied in our previous analysis of a five-dimensional scenario [12]. The two extra dimensions are assumed to be compactified on a torus with radii $R_{1}$ and $R_{2}$. A point in the six-dimensional space is denoted as $\left(x^{\mu}, y_{1}, y_{2}\right)$, where $x^{\mu}$ stands for ordinary spacetime coordinates $(\mu=0, \ldots, 3)$ and $y_{1(2)} \sim y_{1(2)}+2 \pi R_{1(2)}$ are periodic coordinates on the torus.

As mentioned above, the scalar field $(\Phi)$ causing the localization is supposed to form two vortices and two antivortices. A vortex solution is given by $\Phi=e^{i \theta} \phi(r)$, with $\phi(0)=0$ and $\phi(r=$ large $) \simeq$ const., where $r$ is a radial coordinate [17]. For antivortices one simply changes the sign of $\theta$. As will be seen soon, neutrinos are trapped inside a vortex, the throat of which can be viewed as a four-dimensional brane.

The neutrino sector of our model consists of six-dimensional chiral spinors, a lefthanded spinor $\Psi_{1}$ and a right-handed spinor $\Psi_{2}$, both associated with active neutrinos, together with a spinor $N$, which is associated with an inert neutrino and which, as will 
be seen later, can be chosen right-handed. The fields described by the spinors $\Psi_{1}$ and $\Psi_{2}$ are confined to branes or mirror branes, whereas $N$ exists everywhere in the bulk. This choice is a minimal set-up needed to build a successful model on a torus. However, for technical reasons we will keep for a while both chiral components of the spinor fields in our considerations. Contrary to the five-dimensional case [12, two active fields have now to be introduced, in order to accomplish the localization of the fields.

The six-dimensional action of our model is taken to be

$$
\begin{aligned}
S= & \int d^{4} x d^{2} y\left[i \bar{\Psi}_{1} \Gamma^{A} \partial_{A} \Psi_{1}+i \bar{\Psi}_{2} \Gamma^{A} \partial_{A} \Psi_{2}+\left(g \Phi \bar{\Psi}_{1} \Psi_{2}+\text { h.c. }\right)\right. \\
& \left.+i \bar{N} \Gamma^{A} \partial_{A} N+\left(\kappa H^{*} \bar{N} \Psi_{1}+\text { h.c. }\right)\right],
\end{aligned}
$$

where $A=0, \ldots, 5, \kappa$ is a dimensionful Yukawa coupling, and $H$ corresponds to the ordinary Higgs field. The gamma matrices read

$$
\Gamma^{\mu}=\left(\begin{array}{cc}
0 & \gamma^{\mu} \\
\gamma^{\mu} & 0
\end{array}\right), \quad \Gamma^{4}=\left(\begin{array}{cc}
0 & 1_{4} \\
-1_{4} & 0
\end{array}\right), \quad \Gamma^{5}=-i\left(\begin{array}{cc}
0 & \gamma^{5} \\
\gamma^{5} & 0
\end{array}\right)
$$

where $\gamma^{\mu}$ 's are the usual four-dimensional gamma matrices, and $\gamma^{5}=i \gamma^{0} \gamma^{1} \gamma^{2} \gamma^{3}$. It is easy to show that $\Gamma^{A}$ 's fulfil the six-dimensional Clifford algebra $\left\{\Gamma^{A}, \Gamma^{B}\right\}=2 g^{A B} I_{8}$, with $g^{A B}=\operatorname{diag}(1,-1,-1,-1,-1,-1)$. Furthermore, by defining the six-dimensional chirality operators, $P_{ \pm}=\frac{1}{2}\left(I_{8} \pm \Gamma^{7}\right)$, where $\Gamma^{7}=-\Gamma^{0} \Gamma^{1} \cdots \Gamma^{5}=\operatorname{diag}\left(1_{4},-1_{4}\right)$, one is able to decompose the six-dimensional spinors as $\Psi_{1}=\left(\Psi_{1+} \Psi_{1-}\right)^{T}$ and similarly for $\Psi_{2}$ and $N^{1}$. The subscript + denotes a right-handed and the subscript - a left-handed spinor component in the six-dimensional sense.

The reader should note that we have not presented the gauge boson contributions, free scalar parts, nor the non-neutrino (fermion) parts in the action (11), but only the parts essential to read out the neutrino couplings. Gauge bosons are, as mentioned earlier in this paper, trapped to the vortices, so that, effectively, each vortex has its own SM gauge bosons, which couple directly only to fermions on the very same brane. Free scalar parts, on the other hand, are the standard ones, and the non-neutrino parts are out of the scope of the present paper.

\footnotetext{
${ }^{1}$ See e.g. Ref. [19] for an earlier discussion on six-dimensional diracology, chiralities in six and four dimensions, and other related issues.
} 
Let us further point out some peculiar features of our action. The form of the action (11) follows from the imposition of a global U(1) symmetry, under which the fields $\Psi_{1}$, $\Psi_{2}, \Phi, N$ and $H$ are taken to have quantum numbers 1, -1, 2, 0 and 1, respectively. Especially, as will be seen below, the term $\Phi \bar{\Psi}_{1} \Psi_{2}$ takes care of the localization of the active neutrinos on the branes. Terms like $\Phi \bar{N} N, \Phi \bar{\Psi}_{i} \Psi_{i}, \bar{\Psi}_{i} \Psi_{j}(i \neq j), H^{*} \bar{N} \Psi_{2}$ and $H \bar{N} \Psi_{1}$ are forbidden by the U(1) symmetry. Mass terms $\bar{\Psi}_{i} \Psi_{i}$ and $\bar{N} N$ as well as the bulk neutrino Majorana mass term $\sim \bar{N} N^{c}$, allowed by the symmetry, do not survive as we will put $\Psi_{1+}=\Psi_{2-}=N_{-}=0$ later on. Combinations $\Phi^{(*)} \bar{\Psi}_{1(2)} \Psi_{1(2)}^{c}, \bar{\Psi}_{i} \Psi_{j}^{c}(i \neq j)$ and $H \bar{N} \Psi_{2}$, on the other hand, vanish when the $\mathrm{SU}(2)$ structure is taken into account: $\Psi_{i}$ 's and $H$ are the neutral parts of $\mathrm{SU}(2)$ doublets whereas $N$ is a $\mathrm{SU}(2)$ singlet.

We start to analyze our model by studying the localization of the brane fermions. The equations of motion of the fields $\Psi_{1,2}$ are

$$
\begin{aligned}
i \Gamma^{A} \partial_{A} \Psi_{1}+g \Phi \Psi_{2} & =0, \\
i \Gamma^{A} \partial_{A} \Psi_{2}+g^{*} \Phi^{*} \Psi_{1} & =0,
\end{aligned}
$$

where a term $\kappa^{*} H N$ has been neglected, since it may be regarded as a small perturbation in the lowest order approximation. Using polar coordinates $\left(y_{1}=r \cos \theta, y_{2}=r \sin \theta\right)$, one has

$$
\Gamma^{4} \partial_{4}+\Gamma^{5} \partial_{5}=\left(\begin{array}{cc}
0 & e^{-i \gamma^{5} \theta} \partial_{r}-\frac{i}{r} \gamma^{5} e^{-i \gamma^{5} \theta} \partial_{\theta} \\
-e^{i \gamma^{5} \theta} \partial_{r}-\frac{i}{r} \gamma^{5} e^{i \gamma^{5} \theta} \partial_{\theta} & 0
\end{array}\right),
$$

and thus Eqs. (3) can be written in terms of the chiral components of $\Psi_{1,2}$ as

$$
\begin{aligned}
-i \gamma^{\mu} \partial_{\mu} \Psi_{1-} & =i e^{-i \gamma^{5} \theta} \partial_{r} \Psi_{1-}+\frac{1}{r} \gamma^{5} e^{-i \gamma^{5} \theta} \partial_{\theta} \Psi_{1-}+g e^{i \theta} \phi(r) \Psi_{2+}, \\
-i \gamma^{\mu} \partial_{\mu} \Psi_{1+} & =-i e^{i \gamma^{5} \theta} \partial_{r} \Psi_{1+}+\frac{1}{r} \gamma^{5} e^{i \gamma^{5} \theta} \partial_{\theta} \Psi_{1+}+g e^{i \theta} \phi(r) \Psi_{2-} \\
-i \gamma^{\mu} \partial_{\mu} \Psi_{2-} & =i e^{-i \gamma^{5} \theta} \partial_{r} \Psi_{2-}+\frac{1}{r} \gamma^{5} e^{-i \gamma^{5} \theta} \partial_{\theta} \Psi_{2-}+g^{*} e^{-i \theta} \phi(r) \Psi_{1+} \\
-i \gamma^{\mu} \partial_{\mu} \Psi_{2+} & =-i e^{i \gamma^{5} \theta} \partial_{r} \Psi_{2+}+\frac{1}{r} \gamma^{5} e^{i \gamma^{5} \theta} \partial_{\theta} \Psi_{2+}+g^{*} e^{-i \theta} \phi(r) \Psi_{1-}
\end{aligned}
$$

Here we consider the localization on a vortex (i.e. $\Phi=e^{i \theta} \phi(r)$ ); the case of an antivortex localization proceeds along the same lines, with just the chiralities appropriately reversed (see below). 
At this point we return to our original chiral picture by setting $\Psi_{1+}=\Psi_{2-}=0$. Looking at the first and fourth of equations of $\left.(5)^{2}\right)^{2}$, it is now natural to require that $\gamma^{5} \Psi_{1-}=-\Psi_{1-}$ and $\gamma^{5} \Psi_{2+}=-\Psi_{2+}$. This means that $\Psi_{1-}=\frac{1}{2}\left(1-\gamma^{5}\right) \Psi_{1-} \equiv \Psi_{1--}$, the second sign in the subscript referring to the ordinary four-dimensional chirality of $\Psi_{1}$ (i.e., $\Psi_{1}$ is left-handed in four-dimensional sense). Similarly $\Psi_{2+}=\Psi_{2+-}$, i.e., the four-dimensional chirality of $\Psi_{2}$ is left-handed, while its six-dimensional chirality is "righthanded". Furthermore, a solution of the equations of motion apparently exists if $\partial_{\theta} \Psi_{1-}=$ $\partial_{\theta} \Psi_{2+}=0$. Eqs. (15) now reduce to

$$
\begin{aligned}
-i \gamma^{\mu} \partial_{\mu} \Psi_{1--} & =e^{i \theta}\left(i \partial_{r} \Psi_{1--}+g \phi(r) \Psi_{2+-}\right), \\
-i \gamma^{\mu} \partial_{\mu} \Psi_{2+-} & =e^{-i \theta}\left(-i \partial_{r} \Psi_{2+-}+g^{*} \phi(r) \Psi_{1--}\right) .
\end{aligned}
$$

Writing $\Psi_{1--}=\varphi_{1--}(x) f_{1}(r)$ and $\Psi_{2+-}=\varphi_{2+-}(x) f_{2}(r)$, where $f_{1,2}$ are real functions, and $\varphi_{1--}=v \varphi_{2+-}$, one ends up with the equations

$$
\begin{aligned}
-f_{1}(r)\left(i \gamma^{\mu} \partial_{\mu} \varphi_{1--}\right) & =e^{i \theta} \varphi_{1--}\left(i \partial_{r} f_{1}(r)+g v^{-1} \phi(r) f_{2}(r)\right), \\
-v^{-1} f_{2}(r)\left(i \gamma^{\mu} \partial_{\mu} \varphi_{1--}\right) & =e^{-i \theta} \varphi_{1--}\left(-i v^{-1} \partial_{r} f_{2}(r)+g^{*} \phi(r) f_{1}(r)\right) .
\end{aligned}
$$

The 1.h.s. of these equations vanish due to the usual four-dimensional Dirac equation for a massless field. If we take $v= \pm i e^{i \arg (g)}$, the r.h.s. can be easily shown to have solutions behaving like $f_{1,2}(r) \sim \exp \left( \pm|g| \int_{0}^{r} d s \phi(s)\right)$, where, in order to confine $f_{1,2}(r)$ to the vicinity of $r=0$, the plus (minus) sign must be chosen for $\phi(r)<0(>0)$. This concludes our demonstration of the trapping of the spinor fields $\Psi_{1}$ and $\Psi_{2}$ on a vortex by the scalar field $\Phi$.

Two comments are worthwhile at this stage. First, it is straightforward to see what will happen in the antivortex case. Substituting $\Phi=e^{-i \theta} \phi(r)$ to Eqs. (3) and (5), one sees immediately that now a natural choice is $\gamma^{5} \Psi_{1-(2+)}=+\Psi_{1-(2+)}$, and it is hence the right-handed components $\Psi_{1-+}$ and $\Psi_{2++}$ that get localized on an antivortex. This is of course what one would have intuitively expected to happen a priori. Second, as can be

\footnotetext{
${ }^{2}$ The second and third equation would not have entered at all if we had used chiral fields from the beginning.

${ }^{3}$ This requires that $f_{1}(0)=f_{2}(0)$.
} 
deduced from the second and third of equations of (15), the condition $\Psi_{1+}=\Psi_{2-}=0$ not only makes life easier, but is necessary if only the left-handed neutrinos (ordinary active neutrinos) are wanted to get trapped on a vortex and right-handed neutrinos (active mirror neutrinos) on an antivortex. As is known, there is so far no indication of the existence of mirror neutrinos in our four-dimensional world [20].

One may now write somewhat symbolically

$$
\begin{aligned}
\Psi_{1}= & \sqrt{\delta(\bar{y})}\left(\begin{array}{c}
0 \\
\varphi_{1--}(x)
\end{array}\right)+\sqrt{\delta\left(\bar{y}-\bar{y}_{3}\right)}\left(\begin{array}{c}
0 \\
\varphi_{3--}(x)
\end{array}\right)+\sqrt{\delta\left(\bar{y}-\bar{y}_{2}\right)}\left(\begin{array}{c}
0 \\
\varphi_{2-+}(x)
\end{array}\right) \\
& +\sqrt{\delta\left(\bar{y}-\bar{y}_{4}\right)}\left(\begin{array}{c}
0 \\
\varphi_{4-+}(x)
\end{array}\right), \\
\Psi_{2}= & \sqrt{\delta(\bar{y})}\left(\begin{array}{c}
\varphi_{1--}(x) \\
0
\end{array}\right)+\sqrt{\delta\left(\bar{y}-\bar{y}_{3}\right)}\left(\begin{array}{c}
\varphi_{3--}(x) \\
0
\end{array}\right)+\sqrt{\delta\left(\bar{y}-\bar{y}_{2}\right)}\left(\begin{array}{c}
\varphi_{2-+}(x) \\
0
\end{array}\right) \\
& +\sqrt{\delta\left(\bar{y}-\bar{y}_{4}\right)}\left(\begin{array}{c}
\varphi_{4-+}(x) \\
0
\end{array}\right),
\end{aligned}
$$

where the branes (vortices) are situated at $\bar{y}_{1}(=0)$ and $\bar{y}_{3}$, and the mirror branes (antivortices) at $\bar{y}_{2}$ and $\bar{y}_{4}$. The form of $\Psi_{2}$ is due to the requirement that (see above) $\Psi_{1-}(r=0)=\Psi_{2+}(r=0)$ modulo a phase factor that can be absorbed to $\Phi$ (or alternatively to $g$ ) in the original action of Eq. (11). The issue of the Higgs field is now more involved than in the five-dimensional case [12], and the global behavior of the field is not known in general. However, since $H$ can be locally shown to get trapped on a vortex or an antivortex 2, we write

$$
H=\sqrt{\delta(\bar{y})} h_{1}(x)+\sqrt{\delta\left(\bar{y}-\bar{y}_{2}\right)} h_{2}(x)+\sqrt{\delta\left(\bar{y}-\bar{y}_{3}\right)} h_{3}(x)+\sqrt{\delta\left(\bar{y}-\bar{y}_{4}\right)} h_{4}(x)
$$

where $h_{i}$ can be viewed as the vev of the Higgs field in the $i$ th brane. By substituting Eqs. (8) and (9), together with a Kaluza-Klein expansion

$$
\left(\begin{array}{c}
N_{+} \\
N_{-}
\end{array}\right)=\frac{1}{2 \pi \sqrt{R_{1} R_{2}}} \sum_{k, l=-\infty}^{\infty}\left(\begin{array}{c}
N_{+}^{k l}(x) \\
N_{-}^{k l}(x)
\end{array}\right) e^{i k y_{1} / R_{1}+i l y_{2} / R_{2}},
$$

to Eq. (11), one obtains

$$
S=\int d^{4} x\left\{i 2 \bar{\varphi}_{1--} \gamma^{\mu} \partial_{\mu} \varphi_{1--}+i 2 \bar{\varphi}_{2-+} \gamma^{\mu} \partial_{\mu} \varphi_{2-+}+i 2 \bar{\varphi}_{3--} \gamma^{\mu} \partial_{\mu} \varphi_{3--}\right.
$$




$$
\begin{aligned}
& +i 2 \bar{\varphi}_{4-+} \gamma^{\mu} \partial_{\mu} \varphi_{4-+}+\sum_{k, l=-\infty}^{\infty}\left[i \bar{N}_{+}^{k l} \gamma^{\mu} \partial_{\mu} N_{+}^{k l}\right. \\
& +\bar{N}_{++}^{k l}\left(\frac{k}{R_{1}}-\frac{i l}{R_{2}}\right) N_{+-}^{k l}+\bar{N}_{+-}^{k l}\left(\frac{k}{R_{1}}+\frac{i l}{R_{2}}\right) N_{++}^{k l} \\
& +u\left(h_{1}^{*} \bar{N}_{++}^{k l} \varphi_{1--}+h_{2}^{*} z_{2}^{k l} \bar{N}_{+-}^{k l} \varphi_{2-+}\right. \\
& \left.\left.\left.+h_{3}^{*} z_{3}^{k l} \bar{N}_{++}^{k l} \varphi_{3--}+h_{4}^{*} z_{4}^{k l} \bar{N}_{+-}^{k l} \varphi_{4-+}+\text { h.c. }\right)\right]\right\},
\end{aligned}
$$

where

$$
u=\frac{\kappa}{2 \pi \sqrt{R_{1} R_{2}}},
$$

$\kappa$ is taken to be real, and $z_{j}^{k l}=e^{-i k \vartheta_{j}-i l \omega_{j}},\left(\vartheta_{j}, \omega_{j}\right)$ indicating the position of the $j$ th brane in the extra dimensions (with $\vartheta_{1}=\omega_{1}=0$ ). Our analysis complies with a "minimalistic" view in the sense that we have set $N_{-}=0$, i.e., $N$ is taken to be a right-handed chiral field. We remind that this choice made the Dirac and Majorana bulk neutrino mass terms to disappear in the original action (11).

In order to integrate out the massive Kaluza-Klein modes, we proceed along the same lines as we followed in our previous study [12]. This time, however, the ensuing equations are quite lengthy and intransparent and they are mostly omitted in the following presentation. The first ten $k, l$-dependent terms of the action (11) disappear due to the equations of motion of the Kaluza-Klein excitations $N_{+}^{k l}$, for example,

$$
N_{++}^{k l}=-\left(\frac{k}{R_{1}}+\frac{i l}{R_{2}}\right)^{-1}\left(i \gamma^{\mu} \partial_{\mu} N_{+-}^{k l}+u h_{2}^{*} z_{2}^{k l} \varphi_{2-+}+u h_{4}^{*} z_{4}^{k l} \varphi_{4-+}\right)
$$

and similarly for $N_{+-}^{k l}$ (here obviously $(k, l) \neq(0,0)$ ). Substituting these twice to the remaining four h.c.-terms of the action, neglecting higher order derivatives of the bulk fields, and regarding $h_{i}$ 's as constants, one obtains

$$
\begin{aligned}
\mathcal{L}_{(k, l) \neq(0,0)}= & -u^{2} R_{1}\left(\Xi_{\vartheta}\left(\varphi_{i}\right)+h . c .\right)+i u^{2} \rho R_{1}\left(\Xi_{\omega}\left(\varphi_{i}\right)-h . c .\right) \\
& +i u^{2} R_{1}^{2}\left[\left|h_{1}\right|^{2} s(0,0, \rho) \bar{\varphi}_{1--} \gamma^{\mu} \partial_{\mu} \varphi_{1--}+\left|h_{2}\right|^{2} s(0,0, \rho) \bar{\varphi}_{2-+} \gamma^{\mu} \partial_{\mu} \varphi_{2-+}\right. \\
& +\left|h_{3}\right|^{2} s(0,0, \rho) \bar{\varphi}_{3--} \gamma^{\mu} \partial_{\mu} \varphi_{3--}+\left|h_{4}\right|^{2} s(0,0, \rho) \bar{\varphi}_{4-+} \gamma^{\mu} \partial_{\mu} \varphi_{4-+} \\
& +\left(h_{1} h_{3}^{*} s\left(\vartheta_{31}, \omega_{31}, \rho\right) \bar{\varphi}_{1--} \gamma^{\mu} \partial_{\mu} \varphi_{3--}\right. \\
& \left.\left.+h_{2} h_{4}^{*} s\left(\vartheta_{42}, \omega_{42}, \rho\right) \bar{\varphi}_{2-+} \gamma^{\mu} \partial_{\mu} \varphi_{4-+}+h . c .\right)\right]
\end{aligned}
$$


where $\vartheta_{i j}=\vartheta_{i}-\vartheta_{j}, \omega_{i j}=\omega_{i}-\omega_{j}$,

$$
\begin{aligned}
s_{\vartheta(\omega)}(\vartheta, \omega, \rho)= & \sum_{(k, l) \neq(0,0)} \frac{k(l)}{k^{2}+\rho^{2} l^{2}} e^{-i k \vartheta-i l \omega}, \\
s(\vartheta, \omega, \rho)= & \sum_{(k, l) \neq(0,0)} \frac{1}{k^{2}+\rho^{2} l^{2}} e^{-i k \vartheta-i l \omega}, \\
\Xi_{\omega(\vartheta)}\left(\varphi_{i}\right)= & (\overline{+}) h_{2} h_{1}^{*} s_{\vartheta(\omega)}\left(\vartheta_{12}, \omega_{12}, \rho\right) \bar{\varphi}_{2-+} \varphi_{1--} \\
& (\overline{+}) h_{4} h_{1}^{*} s_{\vartheta(\omega)}\left(\vartheta_{14}, \omega_{14}, \rho\right) \bar{\varphi}_{4-+} \varphi_{1--} \\
& +h_{3} h_{2}^{*} s_{\vartheta(\omega)}\left(\vartheta_{23}, \omega_{23}, \rho\right) \bar{\varphi}_{3--} \varphi_{2-+} \\
& (\overline{+}) h_{4} h_{3}^{*} s_{\vartheta(\omega)}\left(\vartheta_{34}, \omega_{34}, \rho\right) \bar{\varphi}_{4-+} \varphi_{3--},
\end{aligned}
$$

and $\rho=R_{1} / R_{2}$. Combining then Eqs. (14) and (11) (with $k=l=0$ ), we end up with the effective Lagrangian

$$
\begin{aligned}
\mathcal{L}_{e f f}= & i \bar{\varphi}_{1} \gamma^{\mu} \partial_{\mu} \varphi_{1}+i \bar{\varphi}_{2} \gamma^{\mu} \partial_{\mu} \varphi_{2}+i \bar{\varphi}_{3} \gamma^{\mu} \partial_{\mu} \varphi_{3}+i \bar{\varphi}_{4} \gamma^{\mu} \partial_{\mu} \varphi_{4}+i \bar{N}_{+} \gamma^{\mu} \partial_{\mu} N_{+} \\
& +u\left(h_{1}^{*} n_{1} \bar{N}_{++} \varphi_{1}+h_{2}^{*} n_{2} \bar{N}_{+-} \varphi_{2}+h_{3}^{*} n_{3} \bar{N}_{++} \varphi_{3}+h_{4}^{*} n_{4} \bar{N}_{+-} \varphi_{4}+\text { h.c. }\right) \\
& -u^{2} R_{1}\left(\Xi_{\vartheta}\left(n_{i} \varphi_{i}\right)+\text { h.c. }\right)+i u^{2} \rho R_{1}\left(\Xi_{\omega}\left(n_{i} \varphi_{i}\right)-\text { h.c. }\right) \\
& +i\left(\tilde{a} \bar{\varphi}_{1} \gamma^{\mu} \partial_{\mu} \varphi_{3}+\tilde{b} \bar{\varphi}_{2} \gamma^{\mu} \partial_{\mu} \varphi_{4}+\text { h.c. }\right)
\end{aligned}
$$

where we have suppressed the chirality indices of $\varphi_{i}$ 's and the Kaluza-Klein indices of $N^{\prime}$ 's, rescaled $\varphi_{i}$ fields with $n_{i}=\left(2+u^{2} R_{1}^{2}\left|h_{i}\right|^{2} s(0,0, \rho)\right)^{-1 / 2}$, and we have denoted

$$
\begin{aligned}
\tilde{a} & =u^{2} R_{1}^{2} h_{1} h_{3}^{*} n_{1} n_{3} s\left(\vartheta_{31}, \omega_{31}, \rho\right) \\
\tilde{b} & =u^{2} R_{1}^{2} h_{2} h_{4}^{*} n_{2} n_{4} s\left(\vartheta_{42}, \omega_{42}, \rho\right) .
\end{aligned}
$$

Note that $s(0,0, \rho)$ is formally a divergent function. Therefore physical cut-offs $k<R_{1} M_{*}$, $l<R_{2} M_{*}$ have been introduced in the sums. This leads to the expression

$$
\rho s(0,0, \rho) \simeq 2 \pi \ln \frac{M_{P l}}{M_{*}}+\pi+\frac{\pi^{2}}{3}\left(\rho+\frac{1}{\rho}\right)-\frac{2}{M}\left(\frac{1}{R}_{1}+\frac{1}{R_{2}}\right),
$$

where $M_{*}=\left(M_{P l}^{2} / R_{1} R_{2}\right)^{1 / 4}$. 
Finally, by defining

$$
\left(\begin{array}{l}
\varphi_{1} \\
\varphi_{2} \\
\varphi_{3} \\
\varphi_{4}
\end{array}\right)=\frac{1}{\sqrt{2}}\left(\begin{array}{cccc}
e^{i \zeta_{a}} & 0 & e^{i \zeta_{a}} & 0 \\
0 & e^{i \zeta_{b}} & 0 & e^{i \zeta_{b}} \\
-e^{-i \zeta_{a}} & 0 & e^{-i \zeta_{a}} & 0 \\
0 & -e^{-i \zeta_{b}} & 0 & e^{-i \zeta_{b}}
\end{array}\right)\left(\begin{array}{l}
\xi_{1} \\
\xi_{2} \\
\xi_{3} \\
\xi_{4}
\end{array}\right), \zeta_{a}=\frac{1}{2} \arg \tilde{a}, \zeta_{b}=\frac{1}{2} \arg \tilde{b},
$$

in order to get rid of the kinetic cross terms, and rescaling $\xi_{i}$ 's with $\tilde{n}_{1(3)}=(1(\overline{+})|\tilde{a}|)^{-1 / 2}$ and $\tilde{n}_{2(4)}=(1(\overline{+})|\tilde{b}|)^{-1 / 2}$, Eq. (16) may be cast into the form

$$
\begin{aligned}
\mathcal{L}_{e f f}= & i \bar{\xi}_{1} \gamma^{\mu} \partial_{\mu} \xi_{1}+i \bar{\xi}_{2} \gamma^{\mu} \partial_{\mu} \xi_{2}+i \bar{\xi}_{3} \gamma^{\mu} \partial_{\mu} \xi_{3}+i \bar{\xi}_{4} \gamma^{\mu} \partial_{\mu} \xi_{4}+i \bar{N}_{+} \gamma^{\mu} \partial_{\mu} N_{+} \\
& -\left(\mathcal{A} \bar{\xi}_{2} \xi_{1}+\mathcal{B} \bar{\xi}_{4} \xi_{1}+\mathcal{C} \bar{\xi}_{2} \xi_{3}+\mathcal{D} \bar{\xi}_{4} \xi_{3}+\text { h.c. }\right) \\
& -\left(\mathcal{E} \bar{N}_{++} \xi_{1}+\mathcal{F} \bar{N}_{+-} \xi_{2}+\mathcal{G} \bar{N}_{++} \xi_{3}+\mathcal{H} \bar{N}_{+-} \xi_{4}+\text { h.c. }\right)
\end{aligned}
$$

where

$$
\begin{aligned}
\mathcal{E} & =-\frac{u \tilde{n}_{1}}{\sqrt{2}}\left(h_{1}^{*} n_{1} e^{i \zeta_{a}}-h_{3}^{*} n_{3} e^{-i \zeta_{a}}\right), \\
\mathcal{F} & =-\frac{u \tilde{n}_{2}}{\sqrt{2}}\left(h_{2}^{*} n_{2} e^{i \zeta_{b}}-h_{4}^{*} n_{4} e^{-i \zeta_{b}}\right), \\
\mathcal{G} & =-\frac{u \tilde{n}_{3}}{\sqrt{2}}\left(h_{1}^{*} n_{1} e^{i \zeta_{a}}+h_{3}^{*} n_{3} e^{-i \zeta_{a}}\right), \\
\mathcal{H} & =-\frac{u \tilde{n}_{4}}{\sqrt{2}}\left(h_{2}^{*} n_{2} e^{i \zeta_{b}}+h_{4}^{*} n_{4} e^{-i \zeta_{b}}\right) .
\end{aligned}
$$

The explicit expressions of the quantities $\mathcal{A}, \mathcal{B}, \mathcal{C}$ and $\mathcal{D}$ are irrelevant for what follows. One should notice that if the phases of $h_{i}$ 's are absorbed to the $\varphi_{i}$ fields in Eq. (11), one has, $s(\vartheta, \omega, \rho)$ being real, $\zeta_{a}=\zeta_{b}=0$, and thus also $\mathcal{E}, \mathcal{F}, \mathcal{G}$ and $\mathcal{H}$ can all be made real.

\section{Neutrino phenomenology of the model}

We now move to consider the phenomenological aspects of our scheme, in particular the effects of the multibrane structure on neutrino physics. Apart from gravitons, only sterile neutrinos can mediate contacts between branes and mirror branes through the bulk, thereby affecting neutrino phenomenology at our home brane. 
From the effective Lagrangian $\mathcal{L}_{\text {eff }}$ in Eq. (20) one infers the following mass Lagrangian for neutrinos:

$$
\mathcal{L}_{M}=-\left(\bar{\xi}_{2} \bar{\xi}_{4} \bar{N}_{++}\right) M\left(\begin{array}{c}
\xi_{1} \\
\xi_{3} \\
N_{+-}
\end{array}\right)+\text {h.c. }
$$

where the matrix $M$ is given by

$$
M=\left(\begin{array}{ccc}
\mathcal{A} & \mathcal{C} & \mathcal{F} \\
\mathcal{B} & \mathcal{D} & \mathcal{H} \\
\mathcal{E} & \mathcal{G} & 0
\end{array}\right)
$$

Even though $M$ can in principle be diagonalized in its general form, this task becomes more straightforward if the branes are assumed to be distributed symmetrically on the torus $^{4}$. We have made this assumption in the following. Then, as can be deduced from Eqs. (15), $\Xi_{\vartheta}=\Xi_{\omega}=0$ and consequently $\mathcal{A}=\mathcal{B}=\mathcal{C}=\mathcal{D}=0$. As will be seen later, even this simplified scenario leads to diverse neutrino phenomenology. Defining

$$
O_{\alpha}=\left(\begin{array}{ccc}
\cos \alpha & \sin \alpha & 0 \\
-\sin \alpha & \cos \alpha & 0 \\
0 & 0 & 1
\end{array}\right), O_{\beta}=\left(\begin{array}{ccc}
\cos \beta & 0 & \sin \beta \\
-\sin \beta & 0 & \cos \beta \\
0 & 1 & 0
\end{array}\right)
$$

with

$$
\cos \alpha=\frac{\mathcal{G}}{\sqrt{\mathcal{E}^{2}+\mathcal{G}^{2}}}, \quad \cos \beta=\frac{\mathcal{H}}{\sqrt{\mathcal{F}^{2}+\mathcal{H}^{2}}},
$$

we obtain

$$
O_{\beta}^{T} M O_{\alpha}=\operatorname{diag}\left(m_{1}, m_{2}, m_{3}\right)
$$

where the eigenvalues are $m_{1}=0, m_{2}=\sqrt{\mathcal{E}^{2}+\mathcal{G}^{2}}$ and $m_{3}=\sqrt{\mathcal{F}^{2}+\mathcal{H}^{2}}$. The corresponding mass eigenstates are

$$
\left(\begin{array}{l}
\chi_{1} \\
\chi_{2} \\
\chi_{3}
\end{array}\right)=O_{\alpha}^{T}\left(\begin{array}{c}
\xi_{1} \\
\xi_{3} \\
N_{+-}
\end{array}\right)+O_{\beta}^{T}\left(\begin{array}{c}
\xi_{2} \\
\xi_{4} \\
N_{++}
\end{array}\right)
$$

The neutrino sector thus consists of one massless and two massive Dirac neutrinos. We emphasize that the existence of one massless eigenstate is not an intrinsic feature of our

\footnotetext{
${ }^{4}$ For example $\vartheta_{3}=\omega_{3}=\vartheta_{2}=\omega_{4}=\pi, \omega_{2}=\vartheta_{4}=0$. Then $s\left(\vartheta_{31}, \omega_{31}, \rho\right)=s\left(\vartheta_{42}, \omega_{42}, \rho\right)=s(\pi, \pi, \rho)$, which is easily calculable numerically.
} 
scenario but results from our choice of symmetrically located branes. Another characteristic feature of the model is the imposed right-handed chirality of the bulk field $N$. As mentioned before, this choice automatically wipes out the six-dimensional Dirac and Majorana bulk neutrino mass terms, thus removing a usual need to rely on ad hoc arguments.

Let us remind the reader that in Eq. (27) $\xi_{1}$ and $\xi_{3}$ are related to left-handed active neutrinos (through e.g. Eq. (19)), $\xi_{2}$ and $\xi_{4}$ to right-handed active mirror neutrinos and the $N$ fields represent sterile bulk neutrinos not confined to any brane. Using Eqs. (19), (24) and (27), taking into account the field rescalings, and normalizing correctly, the original left-handed neutrino of "our" brane is then given by

$$
\varphi_{1}=\cos \theta \chi_{1 L}+\sin \theta \chi_{2 L}
$$

where

$$
\cos \theta=\frac{\tilde{n}_{1} \cos \alpha-\tilde{n}_{3} \sin \alpha}{\sqrt{\tilde{n}_{1}^{2}+\tilde{n}_{3}^{2}}} .
$$

As $\varphi_{1}$ is the only active neutrino living on our brane, only the mixing angle $\theta$ can be experimentally probed. The angle $\beta$ is related to the right-handed fields $\varphi_{2}$ and $\varphi_{4}$, and is therefore a measurable quantity only on the mirror branes.

It should be pointed out that the physical setting of the neutrino mixing differs in one noteworthy respect from the results of our previous five-dimensional study. In [12 the ordinary left-handed neutrino was found to be mixed with an inert left-handed bulk neutrino. Now, as can be seen easily by repeating the calculations similar to those leading to Eq. (28), the other linear combination (not necessarily orthogonal to Eq. (28)) of $\chi_{1 L}$ and $\chi_{2 L}$ is $\varphi_{3} . \varphi_{1}$ is hence mixed with a left-handed active neutrino living on another brane.

Similarly, as seen from Eq. (28), the massless neutrino $\chi_{1}$ and the massive state $\chi_{2}$ appear as mixed in weak interactions. There exist several empirical constraints on their mixing angle $\theta$ and the mass $m_{2}$, coming from laboratory experiments, astrophysical observations and cosmological considerations. In Fig. 1 we compile (as a rough stepfunction approximation sufficient for our purposes) the existing laboratory bounds on 
the mixing angle $\theta$ between the electron neutrino and a heavier neutrino in various mass ranges of the mass $m_{2}$, see Refs. [21]. These bounds follow mainly from the lack of extra kinematical thresholds in the spectra of various particle decays (e.g. beta decay or $\pi^{+} \rightarrow e^{+} \nu_{e}$ ) or the nonobservation of the heavier neutrino decays like $\nu \rightarrow \nu_{e}+e^{-}+e^{+}$. One should take into account also the upper limit of the electron neutrino mass from the tritium beta decay experiment [22],

$$
m_{\nu_{e}} \lesssim 2.2 \mathrm{eV}
$$

This bound should be fulfilled by the mass eigenvalue $m_{2}$, whenever the electron neutrino is predominantly the heavier mass eigenstate $\chi_{2}$. An important constraint at low $m_{2}$ values is given by the oscillation limit from the Bugey disappearance experiment [23]. The upper bound for $\sin ^{2} 2 \theta$ varies in the range 0.02 to 0.3 for $m_{2}^{2}$ in the range $0.01 \mathrm{eV}^{2}$ to $100 \mathrm{eV}^{2}$.

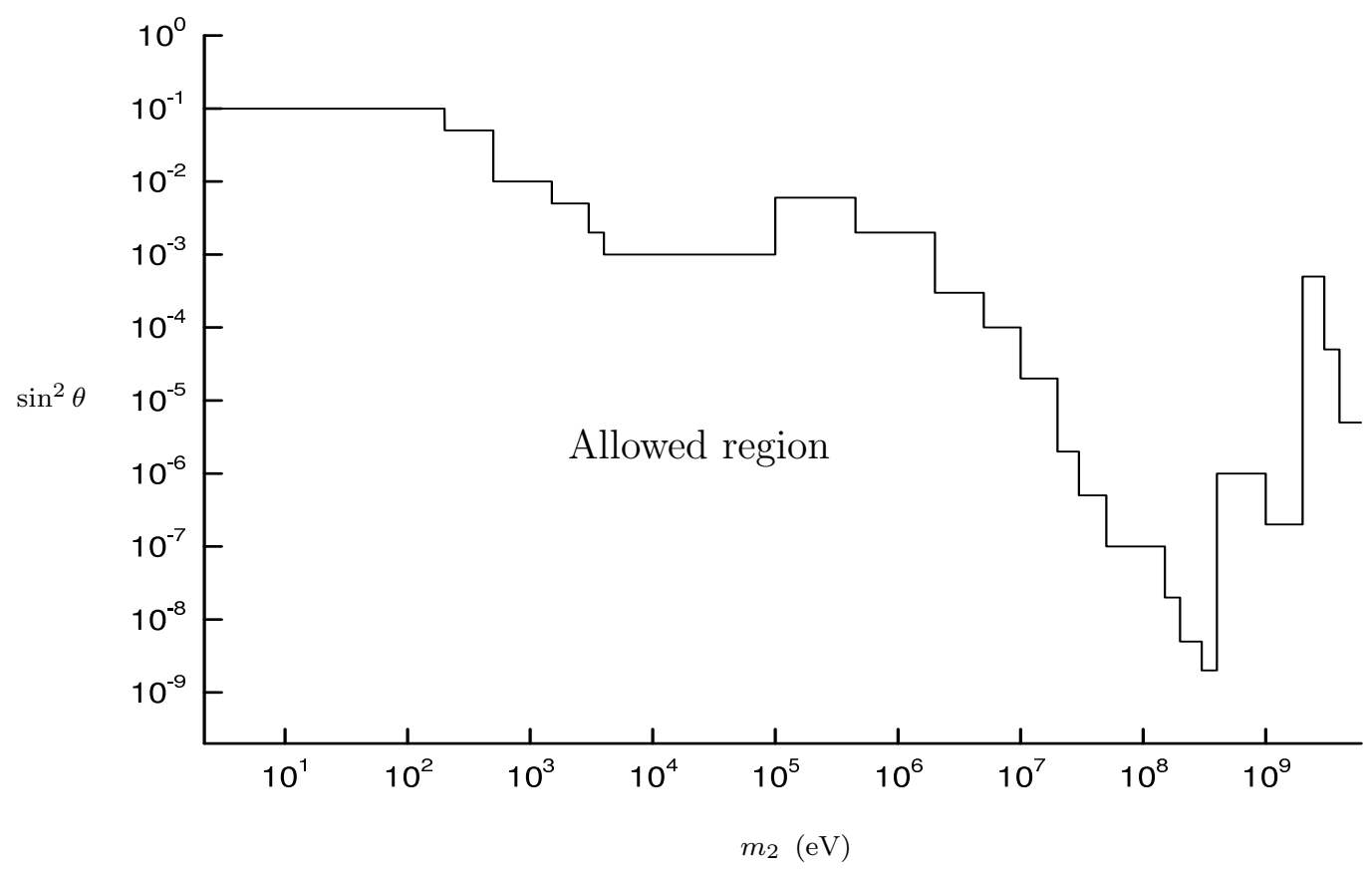

Figure 1: Laboratory bounds on the mixing angle between the electron neutrino and a sterile neutrino from laboratory measurements as a function of the mass of the heavier mass eigenstate (the lighter eigenstate is massless).

The active-sterile neutrino mixing is constrained also by cosmology. If the mixing is too 
large, neutrino oscillations, acting as an effective interaction, would bring sterile neutrinos into equilibrium with the light SM particles before neutrino decoupling. The resulting excess in energy density would endanger the standard scheme of the nucleosynthesis of light elements 24]. This leads to the following bound for $\nu_{e} \leftrightarrow \nu_{s}$ mixing [25]:

$$
\begin{array}{ll}
\left|\delta m^{2}\right| \sin ^{2} 2 \theta<5 \times 10^{-8} \mathrm{eV}^{2} & \text { for }\left|\delta m^{2}\right|<4 \mathrm{eV}^{2}, \\
\sin ^{2} 2 \theta<10^{-8} & \text { for }\left|\delta m^{2}\right|>4 \mathrm{eV}^{2}
\end{array}
$$

(This bound is avoided if there was a suitable net lepton number in the early universe [26.)

We will now confront our scheme with these constraints. We will search the allowed regions of the parameter space of the model numerically using a Monte Carlo analysis. We vary three unknown parameters: the radii of the two extra dimensions $R_{1}$ and $R_{2}$, and the Higgs vacuum expectation value $h_{3}$. The extra dimension radii are varied from the Planck scale to a millimeter scale and the Higgs vev between $10^{-2} \mathrm{MeV}$ and $10^{12} \mathrm{MeV}$. Both $R_{i}$ 's and $h_{3}$ are randomized so that their logarithms are evenly distributed. For $h_{1}$ we use the value $174 \mathrm{GeV}$, and, as can be seen from Secs. 2 and $3, h_{2}$ and $h_{4}$ do not affect neutrino physics on our brane.

In addition to these parameters the action depends on the dimensionful Yukawa coupling $\kappa$. The natural scale for it can be thought to be set by the higher-dimensional Planck scale $M_{*}$, and therefore we write (with $M_{P l}^{2}=M_{*}^{4} R_{1} R_{2}$ )

$$
\kappa=\frac{\kappa^{\prime}}{M_{*}}=\kappa^{\prime}\left(\frac{R_{1} R_{2}}{M_{P l}^{2}}\right)^{1 / 4},
$$

where $\kappa^{\prime}$ is a dimensionless coupling constant. The value of $\kappa^{\prime}$ is not really known, but a plausible choice would be a number relatively close to unity. We have performed our analysis for the values $\kappa^{\prime}=1$ and $\kappa^{\prime}=0.001$.

In Figs. 2 we present scatter plots in the $\left(\bar{R}, m_{2}\right)$-plane (with $\bar{R}=\sqrt{R_{1} R_{2}}$ ) for the two values of the Yukawa coupling $\left(\kappa^{\prime}=0.001\right.$ and 1$)$. These figures consist solely of "raw data", i.e. neither the cosmological constraint, Eq. (31), the limits of Fig. 1, nor the Bugey constraint are taken into account. The allowed parameter space is seen to be quite severely constrained by the mathematical structure of the model. Nevertheless, the characteristic extra-dimensional size may vary from the millimeter scale some 30 orders 
of magnitude all the way down to the Planck scale, and the higher mass eigenvalue $m_{2}$ ranges from $10^{-9} \mathrm{eV}$ to $10^{10} \mathrm{MeV}$, depending on the coupling $\kappa^{\prime}$. The general trend is that small (large) extra-dimensional size corresponds to large (small) mass. Note that the value of $\kappa^{\prime}$ affects the vertical position of the two diagonal borderlines, whereas the wedge-like region below them remains immutable.
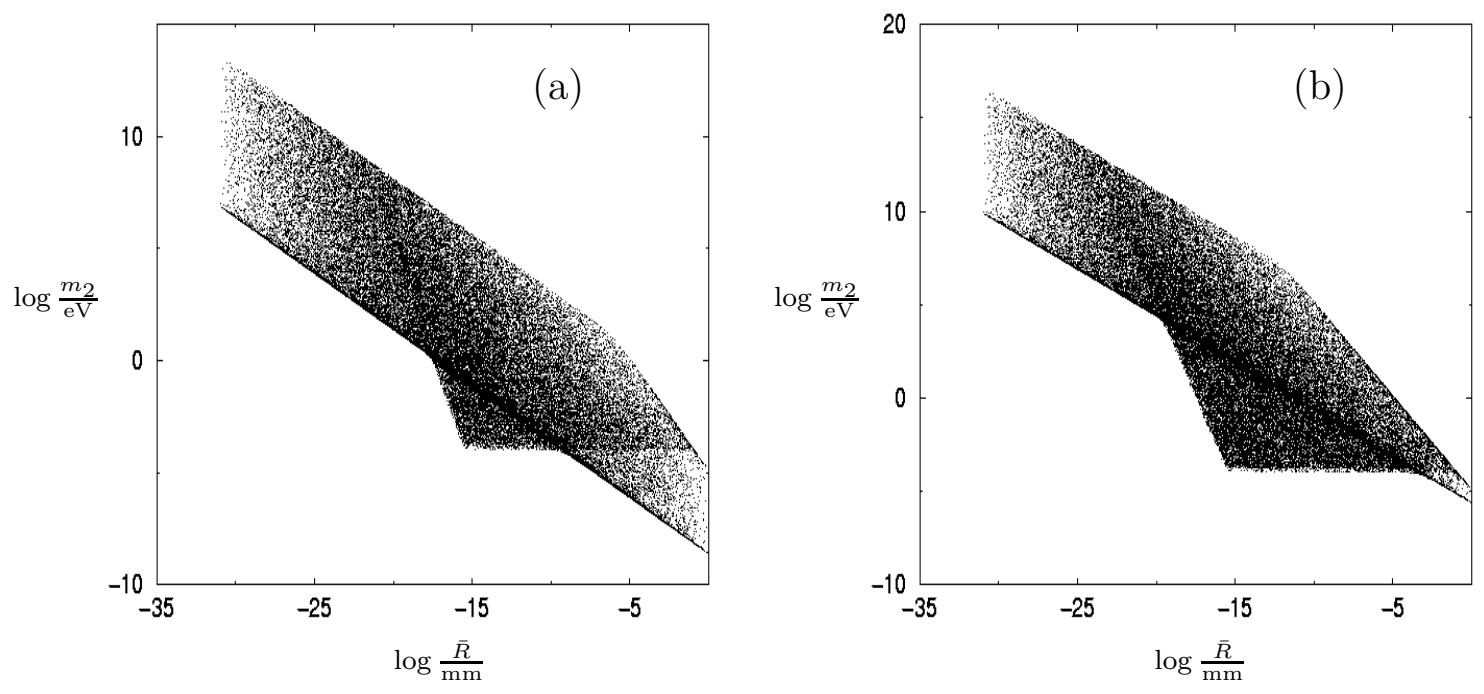

Figure 2: Scattering plot of geometric average of the radii, $\bar{R}$, vs. possible neutrino masses $m_{2}$ without any experimental constraints. a) $\kappa^{\prime}=0.001$, b) $\kappa^{\prime}=1.0$.

Figs. 3 show how the situation changes when the experimental constraints are taken into account. Interestingly, the extreme values on both axes are still allowed and, vaguely speaking, only dots in between are cut out. Numerical investigations suggest that the upper (lower) diagonal borderline reflects the chosen range for the Higgs value $h_{3}$, higher (lower) values enabling mass $m_{2}$ to be still larger (smaller). For the lower diagonal $\sin \theta \approx$ 1. In the case of $\kappa^{\prime}=1$ the allowed region is split up into two separate parts, the feature that will be seen in all the subsequent figures. 

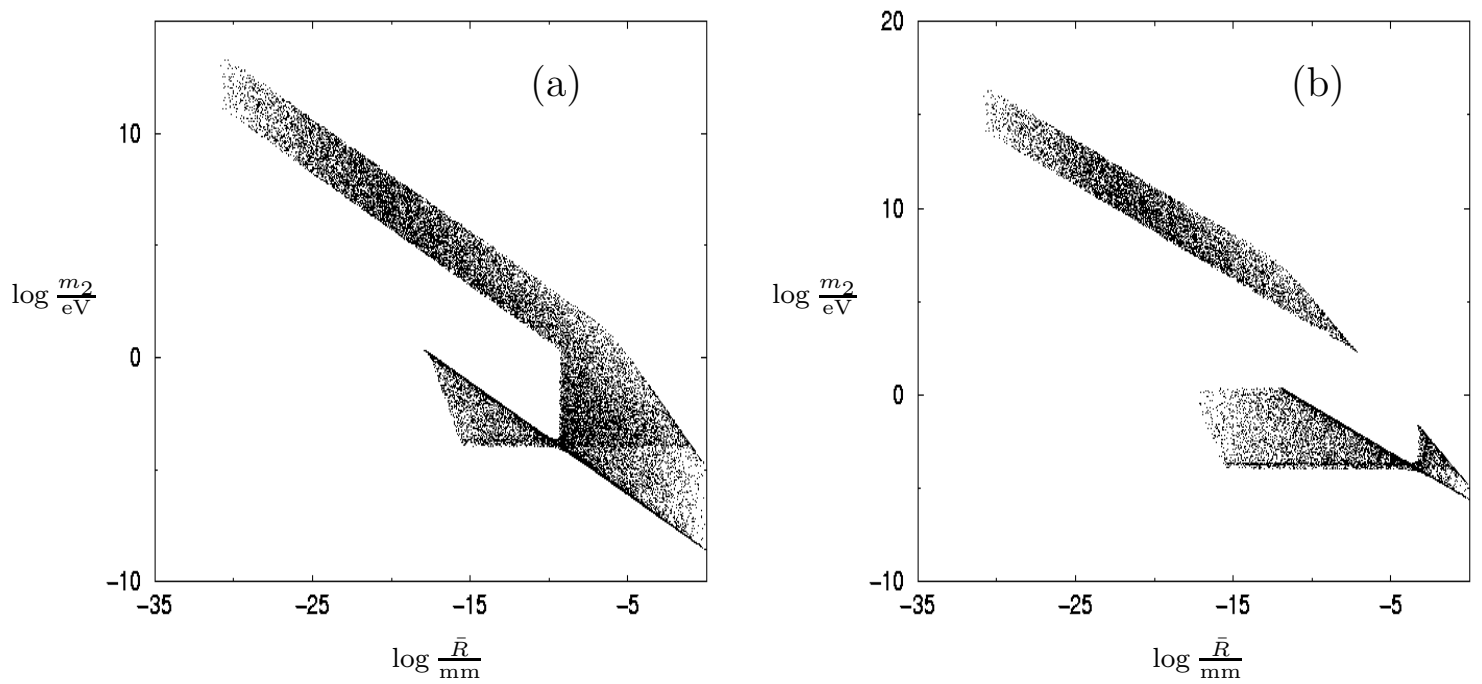

Figure 3: Scattering plot of geometric average of the radii, $\bar{R}$, vs. possible neutrino masses $m_{2}$ with all observational constraints. a) $\kappa^{\prime}=0.001$, b) $\kappa^{\prime}=1.0$.

Figs. 4 display the behavior of the mixing angle $\theta$ as a function of $\bar{R}$. For very small extra-dimensional sizes the mixing is tiny, but above $\bar{R} \sim 10^{-16} \mathrm{~mm}$ maximal mixing, $\theta=\pi / 4$, becomes possible. The peculiarity that $\theta$ can never be exactly zero is a consequence of the mathematical structure of the model, as may be deduced from Eq. (29). However, for larger values of $h_{3}$ the allowed region would extend to smaller angles. The limit just below $\sin \theta=10^{-4}$, on the other hand, is due to the cosmological constraint (31). Note that the obvious change in the scatter plot for $\bar{R} \gtrsim 10^{-10} \mathrm{~mm}\left(\kappa^{\prime}=0.001\right)$ or $\bar{R} \gtrsim 10^{-4} \mathrm{~mm}\left(\kappa^{\prime}=1\right)$, as well as the tilted rightmost borderline penetrating both of the separate parts in the case of $\kappa^{\prime}=1$, have analogous counterparts in Figs. 3.

Finally, in Figs. 5 is shown the allowed parameter space in the $\left(\sin ^{2} 2 \theta, \delta m^{2}\right)$-plane, where $\delta m^{2}=m_{2}^{2}$ as $m_{1}=0$. We have presented in these figures separately those points which correspond to large values of the size $\bar{R}$ of the extra dimension, $0.01 \mathrm{~mm}<\bar{R}<1$ 

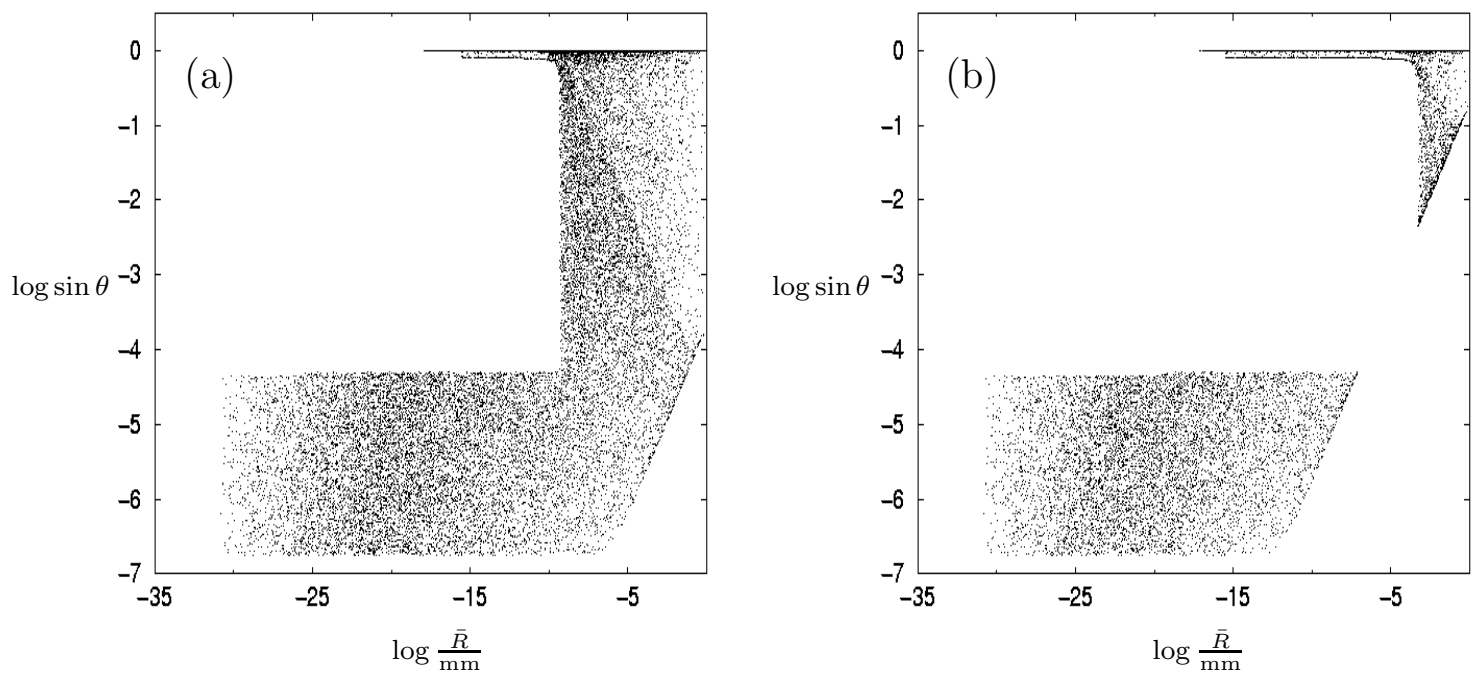

Figure 4: Scattering plot of geometric average of the radii, $\bar{R}$, vs. mixing angle $\sin \theta$ with observational constraints. a) $\kappa^{\prime}=0.001$, b) $\kappa^{\prime}=1.0$.

mm. With $\bar{R}$ in this range, the hierarchy problem can be solved in this model and also the existence of the extra dimensions is testable in the new short-range gravity experiments [5. 27. The empty areas in the large $\sin ^{2} 2 \theta$ large $\delta m^{2}$ region are forbidden by the cosmological constraint (31). The other constraints do not have substantial effects to this plot. As mentioned before, the borderline at small $\theta$ (now at $\sin ^{2} 2 \theta \sim 10^{-13}$ ) corresponds to the upper end of the Higgs vev $h_{3}$ range covered by our Monte Carlo data set, and it would move towards smaller values if the maximum value of the parameter $h_{3}$ is increased.

As can be seen from Figs. 5, our model allows for a vast diversity in the possible $\left(\delta m^{2}, \theta\right)$-combinations. With the chosen ranges of the free parameters of the model, the squared mass difference of the active-sterile neutrino pair can have values from about $10^{-11} \mathrm{eV}^{2}\left(\kappa^{\prime}=1\right)$ or $10^{-17} \mathrm{eV}^{2}\left(\kappa^{\prime}=0.001\right)$ up to $10^{25} \mathrm{eV}^{2}$ or more. However, squared mass differences $\delta m^{2}=m_{2}^{2} \gtrsim 1 \mathrm{eV}^{2}$ are possible only for very small values of the mixing 
angle, $\theta \lesssim 10^{-4}$. If the extra dimension size is in the theoretically and phenomenologically interesting region of $\bar{R} \gtrsim 0.01 \mathrm{~mm}$ (the hits denoted by circles in Figs. 5), $\delta m^{2}$ is forced to much smaller values. In particular, maximal active-sterile mixing is possible in this case only for quite small values of the squared mass difference: $\delta m^{2} \simeq 10^{-11}-10^{-9} \mathrm{eV}^{2}$ for $\kappa^{\prime}=1$ and $\delta m^{2} \simeq 10^{-17}-10^{-15} \mathrm{eV}^{2}$ for $\kappa^{\prime}=0.001$. On the other hand, the largest squared mass difference consistent with the large extra dimension size is of the order of $\delta m^{2} \simeq 10^{-6} \mathrm{eV}^{2}$.

Let us still mention that the leftmost points in the small squared mass difference region, $\delta m^{2} \lesssim 1 \mathrm{eV}^{2}$, correspond to $\theta \approx \pi / 2$, as may be seen particularly well by comparing Figs. $4 \mathrm{~b}$ and $5 \mathrm{~b}$. In this case the predominantly active state, the ordinary neutrino, is the heavier one of the two mass states.
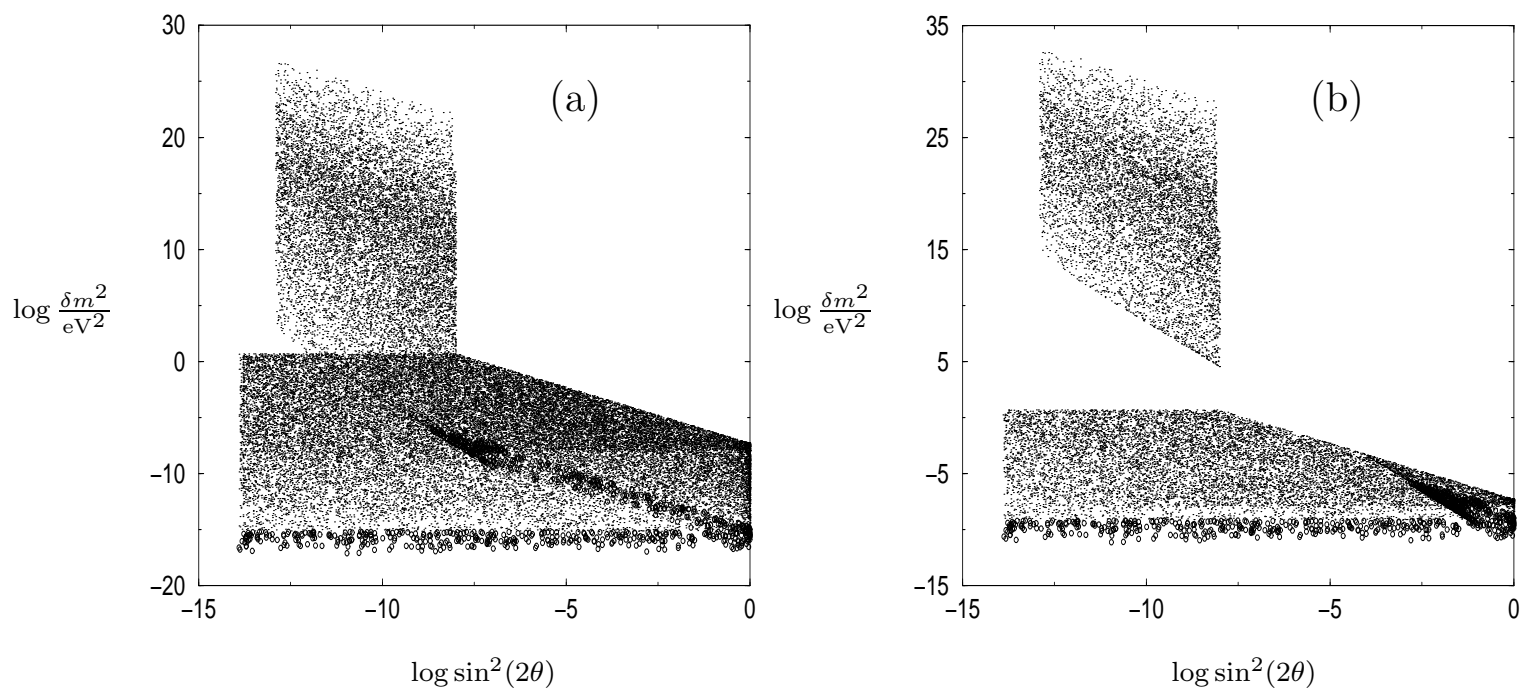

Figure 5: Scattering plot of $\sin ^{2}(2 \theta)$ vs. squared neutrino mass difference $\delta m^{2}$ with experimental and observational constraints taken into account. Circles indicate the points, where $0.01 \mathrm{~mm}<\bar{R}<1 \mathrm{~mm}$. a) $\kappa^{\prime}=0.001$, b) $\kappa^{\prime}=1.0$. 


\section{Summary and conclusions}

We have investigated a six-dimensional model with two extra dimensions compactified on a torus. Particles with Standard Model charges are trapped on four four-dimensional branes (vortices) by a scalar field with an appropriate periodic position dependence on the torus. On two of the branes, one of which is the world we live in, particles have the familiar $V-A$ weak currents, while on the other two, called mirror branes, weak currents are of the $V+A$ form. The structure of the model is mathematically quite strictly determined, but there are some free parameters in the theory such as the size of the extra dimensions, characterized by the radii of the torus $R_{1}$ and $R_{2}$, and the vev $\left(h_{3}\right)$ of the counterparts of the SM Higgs field on the other branes. Also the strength $\kappa^{\prime}$ of the Yukawa coupling between the Higgs field, brane neutrinos and bulk neutrinos, as well as the locations of the vortices on the torus, are not a priori restricted.

Right-handed neutrinos and left-handed mirror neutrinos, which lack Standard Model interactions, can propagate in the extra two-dimensional bulk and thereby mediate mixing between active neutrinos of the branes and mirror branes. This affects neutrino phenomenology on our brane. We have confronted the model with existing data on neutrino masses and active-sterile neutrino mixing from laboratory measurements and cosmology. Our main results can be seen in Figs. 5, where we have plotted the $\left(\sin ^{2} 2 \theta, \delta m^{2}\right)$-parameter space covered by our model when the free parameters of the model are allowed to vary within wide ranges of values. In order the model to solve the hierarchy problem, the size of the extra dimensions should be large enough. The corresponding points in Figs. 5 are denoted by circles, and as one can see, the squared mass difference is in that case forced into a quite narrow range of values, in particular when the mixing is maximal.

We have restricted our phenomenological analysis to the electron neutrino, but conclusions would be very similar for the muon and tau neutrinos, since the most important constraint, the cosmological bound for the active-sterile mixing, is quite insensitive to neutrino flavor.

Let us finally emphasize that the model we have considered is not the most general one but we have made a number of simplifying, albeit conceivable, assumptions. Nevertheless, 
we believe that our analysis has revealed the essential features of the scenario.

\section{Acknowledgements}

This work has been supported by the Academy of Finland under the project no. 40677.

One of us (V.S.) has been funded by the Graduate School of Nuclear and Particle Physics of the Academy of Finland.

\section{References}

[1] G. Nordström, Phys. Z. 15 (1914) 504; T. Kaluza, Sitzungsber. Preuss. Akad. Wiss. Math. Phys. Kl. (1921) 966; O. Klein, Phys. Z. 37 (1926) 895.

[2] N. Arkani-Hamed, S. Dimopoulos and G. Dvali, Phys. Lett. B 429 (1998) 263.

[3] I. Antoniadis, N. Arkani-Hamed, S. Dimopoulos and G. Dvali, Phys. Lett. B 436 (1998) 257.

[4] N. Arkani-Hamed, S. Dimopoulos and G. Dvali, Phys. Rev. D 59 (1999) 086004.

[5] J. C. Long, H. W. Chan and J. C. Price, Nucl. Phys. B 539 (1999) 23.

[6] C. D. Hoyle et al., Phys. Rev. Lett. 86 (2001) 1418.

[7] J. Polchinski, hep-th/9611050.

[8] G. Shiu and S.-H. H. Tye, Phys. Rev. D 58 (1998) 106007.

[9] N. Arkani-Hamed, S. Dimopoulos, G. Dvali and J. March-Russell, Phys. Rev. D 65 (2001) 024032.

[10] K. R. Dienes, E. Dudas and T. Gherghetta, Nucl. Phys. B 557 (1999) 25.

[11] A. E. Faraggi and M. Pospelov, Phys. Lett. B 458 (1999) 237; G. Dvali and A. Yu. Smirnov, Nucl. Phys. B 563 (1999) 63; A. Das and O. C. W. Kong, Phys. Lett. B 470 (1999) 149; R. N. Mohapatra, S. Nandi and A. Perez-Lorenzana, Phys. 
Lett. B 466 (1999) 115; R. N. Mohapatra and A. Perez-Lorenzana, Nucl. Phys. B 576 (2000) 466; G. C. McLaughlin and J. N. Ng, Phys. Lett. B 470 (1999) 157; A. Ioannisian and A. Pilaftsis, Phys. Rev. D 62 (2000) 066001; R. Barbieri, P. Creminelli and A. Strumia, Nucl. Phys. B 585 (2000) 28; A. Lukas, P. Ramond, A. Romanino and G. G. Ross, Phys. Lett. B 495 (2000) 136; A. Ioannisian and J. W. F. Valle, Phys. Rev. D 63 (2001) 073002; R. N. Mohapatra and A. Perez-Lorenzana, Nucl. Phys. B 593 (2001) 451; K. R. Dienes and I. Sarcevic, Phys. Lett. B 500 (2001) 133; K. Agashe and G.-H. Wu, Phys. Lett. B 498 (2001) 230; N. Cosme et al., Phys. Rev. D 63 (2001) 113018; D. O. Caldwell, R. N. Mohapatra and S. J. Yellin, Phys. Rev. Lett. 87 (2001) 041601; D. O. Caldwell, R. N. Mohapatra and S. J. Yellin, Phys. Rev. D 64 (2001) 073001; A. S. Dighe and A. S. Joshipura, Phys. Rev. D 64 (2001) 073012; C. S. Lam and J. N. Ng, Phys. Rev. D 64 (2001) 113006; A. Lukas, P. Ramond, A. Romanino and G. G. Ross, JHEP 04 (2001) 010; J.-M. Frere, M. V. Libanov and S. V. Troitsky, JHEP 11 (2001) 025; C. S. Lam, Phys. Rev. D 65 (2002) 053009; A. de Gouvea, G. F. Giudice, A. Strumia and K. Tobe, Nucl. Phys. B 623 (2002) 395.

[12] J. Maalampi, V. Sipiläinen and I. Vilja, Phys. Lett. B 512 (2001) 91.

[13] V. A. Rubakov and M. E. Shaposhnikov, Phys. Lett. 125B (1983) 136.

[14] N. Arkani-Hamed and M. Schmaltz, Phys. Rev. D 61 (2000) 033005.

[15] R. Jackiw and P. Rossi, Nucl. Phys. B 190 (1981) 681.

[16] K. Akama in Lecture Notes in Physics 176 (Springer-Verlag, 1982), eds. K. Kikkawa et al., p. 267; hep-th/0001113.

[17] H. B. Nielsen and P. Olesen, Nucl. Phys. B 61 (1973) 45.

[18] E. Witten in Modern Kaluza-Klein Theories (Addison-Wesley, 1987), eds. T. Appelquist et al., p. 438.

[19] A. Lukas and A. Romanino, hep-ph/0004130. 
[20] J. Maalampi and M. Roos, Phys. Rept. 186 (1990) 53.

[21] K.-H. Hiddemann, H. Daniel and O. Schwentker, J. Phys. G 21 (1995) 639; E. Holzschuh et al., Phys. Lett. B 451 (1999) 247; E. Holzschuh, L. Palermo, H. Stüssi and P. Wenk, Phys. Lett. B 482 (2000) 1; K. Schreckenbach, G. Colvin and F. von Feilitzsch, Phys. Lett. 129B (1983) 265; J. Deutsch, M. Lebrun and R. Prieels, Nucl. Phys. A 518 (1990) 149; C. Hagner et al., Phys. Rev. D 52 (1995) 1343; D. A. Bryman et al., Phys. Rev. Lett. 50 (1983) 1546; N. De Leener-Rosier et al., Phys. Rev. D 43 (1991) 3611; D. I. Britton et al., Phys. Rev. D 46 (1992) R885; G. Bernardi et al., Phys. Lett. B 203 (1988) 332; J. Dorenbosch et al., Phys. Lett. 166B (1986) 473; C. Akerlof et al., Phys. Rev. D 37 (1988) 577.

[22] J. Bonn et al., Nucl. Phys. Proc. Suppl. 91 (2001) 273.

[23] B. Achkar et al., Nucl. Phys. B 434 (1995) 503.

[24] R. Barbieri and A. Dolgov, Phys. Lett. B 237 (1990) 440 and Nucl. Phys. B 349 (1991) 743; K. Kainulainen, Phys. Lett. B 244 (1990) 191; K. Enqvist, K. Kainulainen and J. Maalampi, Phys. Lett. B 249 (1990) 531 and Nucl. Phys. B 349 (1991) 754 .

[25] X. Shi and G. M. Fuller, Phys. Rev. Lett. 83 (1999) 3120.

[26] R. Foot, M. J. Thomson and R. R. Volkas, Phys. Rev. D 53 (1996) 5349.

[27] J. C. Long, A. B. Churnside and J. C. Price, hep-ph/0009062. 